\title{
Nickel on Lead, Magnetically Dead or Alive?
}

\author{
Go Tateishi and Gerd Bergmann \\ Department of Physics \\ University of Southern California \\ Los Angeles, California 90089-0484 \\ e-mail: bergmann@usc.edu
}

November 2, 2018

\begin{abstract}
Two atomic layers of $\mathrm{Ni}$ condensed onto $\mathrm{Pb}$ films behave, according to anomalous Hall effect measurements, as magnetic dead layers. However, the Ni lowers the superconducting $T_{c}$ of the $\mathrm{Pb}$ film. This has lead to the conclusion that the Ni layers are still very weakly magnetic. In the present paper the electron dephasing due to the Ni has been measured by weak localization. The dephasing is smaller by a factor 100 than the pair-breaking. This proves that the $T_{c}$-reduction in the $\mathrm{PbNi}$ films is not due magnetic Ni moments.
\end{abstract}

PACS: $75.20 . \mathrm{Hr}, 73.20 . \mathrm{Fz}, 74.45 .+\mathrm{c}, 74.78 . \mathrm{Fk}$

When a thin superconductor is condensed onto a normal conducting film then the first layers loose their superconductivity. This phenomenon is called the "superconducting proximity effect". A similar question arises if one condenses a thin ferromagnetic metal onto a normal metal. If the first layers of the ferromagnet loose their magnetism they are denoted as "magnetic dead layers" (MDL). The first dead layers were observed almost 30 years ago for two to three atomic layers of $\mathrm{Ni}$ condensed onto amorphous Bi films [1]. For Ni layers on top of noble metals there were originally contradicting results. Liebermann et al. 2] observed two dead layers of $\mathrm{Ni}$ on $\mathrm{Cu}$ and $\mathrm{Au}$ while Pierce and Siegmann [3] observed ferromagnetism already in mono-layers of $\mathrm{Ni}$ on $\mathrm{Cu}$ (by means of spin polarized photo electrons). Kramer and Bergmann [4] investigated the magnetic properties of $\mathrm{Ni}$ on the surface of $\mathrm{Mg}, \mathrm{In}, \mathrm{Sn}$, and the noble metals $\mathrm{Cu}, \mathrm{Ag}$ and $\mathrm{Au}$ by means of the anomalous Hall effect (AHE). They observed between two and three dead Ni layers on the polyvalent substrate, while Ni on top of the noble metals showed ferromagnetism already for the first Ni mono-layer. However, the electronic properties of the $\mathrm{Ni}$ appeared to be modified for the first two to three $\mathrm{Ni}$ layers because the AHE had the wrong (positive) sign in this range of Ni thickness. Meservey et al. [5], [6] used spin-polarized tunneling to investigate the proximity effect of the ferromagnetic metals. They observed about three dead layers of $\mathrm{Ni}$ on Al. A number of theoretical papers [7], 8], [9], [10], [11], [12], [13] investigated the question of a magnetic proximity effect. 
The occurance of magnetic dead $\mathrm{Ni}$ layers is still a question under investigation [14, [15] while double layers of $\mathrm{PbNi}$ and other pairs of superconductor and ferromagnetic metal experienced a new interest in the superconducting proximity effect [16]. But in this paper we want to address a claim made by Moodera and Meservey (MM) [17], [18], [19], [20], [21], 22] about the properties of single and double layers of $\mathrm{Ni}$ on $\mathrm{Pb}$.

$\mathrm{MM}$ increased the sensitivity in their investigation of $\mathrm{PbNi}$ double layers by using a $9 \mathrm{~nm}$ thick $\mathrm{Pb}$ film as part of $14 \mathrm{MHz}$ oscillator. The frequency of the oscillator changed by about $60 \mathrm{kHz}$ when the $\mathrm{Pb}$ film made a transition from superconducting to normal state. They observed that the deposit of $0.4 \mathrm{~nm}$ of $\mathrm{Ni}$ onto the $\mathrm{Pb}$ substrate reduced the transition temperature of the $\mathrm{Pb}$ below $4.2 \mathrm{~K}$. A similar effect can be produced by the deposition of $\mathrm{Fe}$. However, the pair breaking effect of $\mathrm{Fe}$ is about 80 times stronger than that of Ni.

MM gave their results the following interpretation: The $\mathrm{Ni}$ atoms on top of the $\mathrm{Pb}$ films do not completely loose their moments, even for the smallest coverages, and their magnetic scattering dephases (depairs) the superconducting Cooper pairs, even at their smallest thickness of 0.2 atomic layers of $\mathrm{Ni}$. MM did not try to give a value for this reduced moment.

In this paper we revisit the $\mathrm{PbNi}$ system. We have measured the magnetic scattering of $\mathrm{Ni}$ using the method of weak localization (quantum interference). It is well known and discussed below that the pair-breaking mechanism in superconductivity and the dephasing in weak localization are in many aspects identical. There is, however, an experimental difficulty in measuring weak localization in superconducting $\mathrm{Pb}$ films since the magnetoresistance is overshadowed by the Azlamazov-Larkin fluctuations [23]. Therefore we use only very thin $\mathrm{Pb}$ films between 1 and 10 atomic layers which are condensed onto a $\mathrm{Ag}$ film. Then the proximity effect suppresses the superconductivity of the $\mathrm{Pb}$ layers.

The experimental procedure is the following. A Ag film with a thickness of 35 atomic layers is quench condensed onto a quartz plate at He temperature in an UHV of better than $10^{-11}$ torr. The Ag film is covered in different experiments with $\mathrm{Pb}$ films whose thicknesses lie between one and five atomic layers. Then the $\mathrm{Pb}$ film is covered in several steps with $\mathrm{Ni}$. The first Ag film is chosen for three reasons: (i) Even for quenched condensation it is not possible to condense a homogeneous film of a few atomic layers of $\mathrm{Pb}$ onto a quartz plate. This requires a homogeneous conducting metal film of sufficient thickness as a substrate. (ii) The Ag film suppresses the superconductivity of the extremely thin $\mathrm{Pb}$ film. (iii) $\mathrm{Ni}$ on top of Ag shows magnetism already for a mono-layer of Ni. Therefore the observation of MDLs of $\mathrm{Ni}$ on $\mathrm{AgPb}$ can only be due to the $\mathrm{Pb}$ film in between the $\mathrm{Ag}$ and the $\mathrm{Ni}$. (It also proves that there are no holes in the thin $\mathrm{Pb}$ film).

We use two experimental methods to investigate the magnetic properties of the $\mathrm{AgPbNi}$ multi-layers, (i) the anomalous Hall effect and (ii) weak localization. In Fig.1 the anomalous Hall resistance $R_{y x}(B)$ is plotted as a function of $B$ for $d_{P b}=2$ atomic layers and different Ni thicknesses in a perpendicular magnetic field. The AHE curves can be extrapolated to zero magnetic field, yielding $R_{y x}^{0}$. This AHE resistance $R_{y x}^{0}$ is plotted in Fig.2. It measures the magnetization perpendicular to the film plane in zero magnetic field. The surprising result is that the thickness of the MDL is (almost) independent of the $\mathrm{Pb}$ thickness. Even 
for 1.3 atomic layers of $\mathrm{Pb}$ the $\mathrm{AHE}$ is suppressed up to a $\mathrm{Ni}$ thickness of 2.5 atomic layers The results are collected in table I.
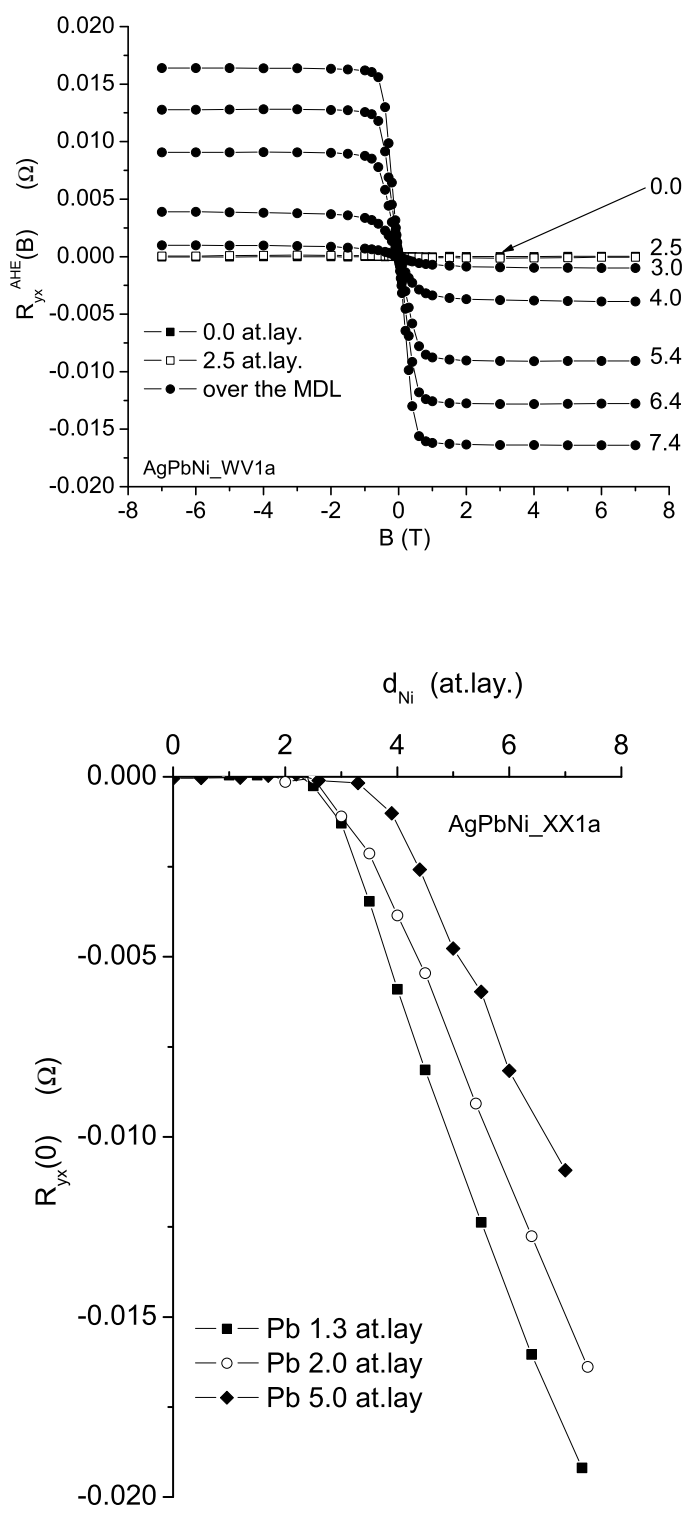

Fig.1: The anomalous Hall curves of an $\mathrm{AgPbNi}$ layer as a function of the Ni thickness.
Fig.2: The anomalous Hall resistance $R_{y x}^{0}$ as a function of the $d_{N i}$ for three different $\mathrm{Pb}$ thicknesses.

\begin{tabular}{|l|l|}
\hline $\mathbf{d}_{\mathbf{P b}}$ & $\mathbf{d}_{\mathbf{M D L}}$ \\
\hline 1.3 & 2.5 \\
\hline 2.0 & 2.5 \\
\hline 5.0 & 3.3 \\
\hline
\end{tabular}

Table I: The number of magnetic dead Ni layers in $\mathrm{AgPbNi}$ multi-layers as measured by the anomalous Hall effect. The first column gives the thickness of the $\mathrm{Pb}$ film in atomic layers

The experimental result confirms in addition that the $\mathrm{Pb}$ film covers the $\mathrm{Ag}$ substrate homogeneously because one would otherwise have regions of magnetic $\mathrm{Ni}$ on $\mathrm{Ag}$ which would be observable. 
The magneto-resistance measurements of weak localization yield the dephasing of the conduction electrons in the multi-layers. In Fig. 3 the magneto-resistance of a $\mathrm{AgPbNi}$ multi-layer with a $\mathrm{Pb}$ thickness of 1.3 atomic layers is plotted for different $\mathrm{Ni}$ coverages. The numbers at the right side of the curves gives the Ni coverage in atomic layers. The open and closed circles are experimental data while the full curves are theoretical fits with two fitting parameters, $H_{i}^{*}$ and $H_{s o}^{*}$.

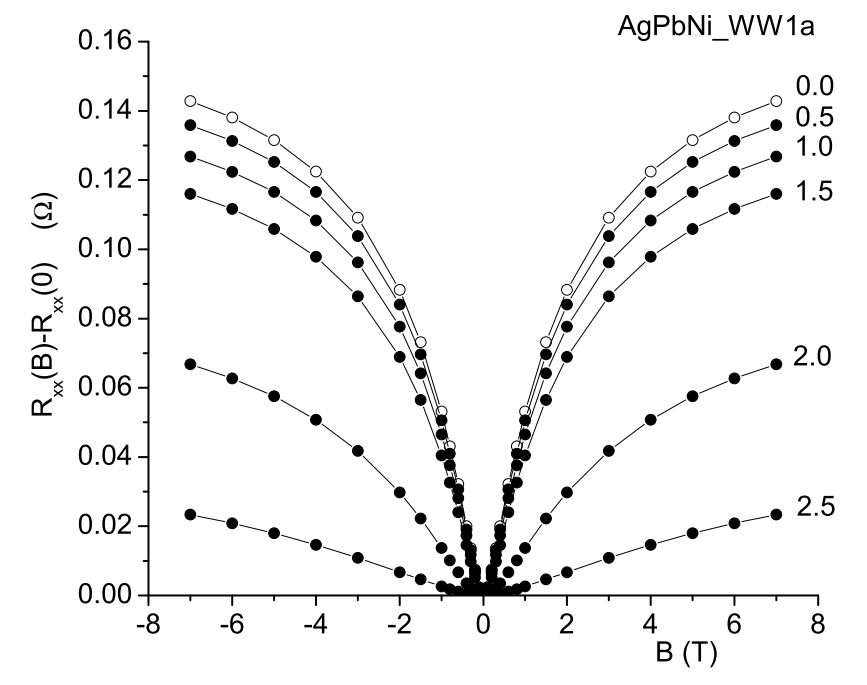

Fig.3: Magneto-resistance of a AgPbNi multi-layer with $d_{P b}=1.3$ atomic layers The open and full circles are experimental data while the full curves are theoretical fits, yielding the singlet and triplet dephasing rates. The numbers at the right of the curves give the Ni thickness in atomic layers.

The theory yields the following expression [24] for the quantum conductance corrections to the resistance

$$
\frac{\Delta R}{R^{2}}=\frac{e^{2}}{2 \pi^{2} \hbar}\left[\frac{1}{2} f\left(\frac{B}{B_{S}}\right)-\frac{3}{2} f\left(\frac{B}{B_{T}}\right)\right]
$$

where $R$ is the resistance per square and $\Delta R / R^{2}=-\Delta G$ is the (negative) change in conductance. The function $f(x)$ is given by

$$
f(x)=\ln (x)+\Psi\left(\frac{1}{2}+\frac{1}{x}\right)
$$

where $\Psi(z)$ is the digamma function and $B_{S}$ and $B_{T}$ are the characteristic fields for singlet and triplet dephasing. They are given by

$$
B_{S}=B_{i}+2 B_{s}=B_{i}^{*} \quad B_{T}=B_{i}+\frac{4}{3} B_{s o}+\frac{2}{3} B_{s}=B_{i}^{*}+\frac{4}{3} B_{s o}^{*}
$$


$B_{i}, B_{s o}, B_{s}$ are the characteristic fields for inelastic, spin-orbit and magnetic scattering. From these fields one can calculate the corresponding scattering times $\tau_{n}$ with the relation

$$
B_{n} \tau_{n}=\frac{\hbar}{4 e D}
$$

where $D$ is the diffusion constant which can be obtained from the resistivity of the layers (see also [25], [26]). This product has a value of $B_{n} \tau_{n} \approx 3 \times 10^{-13}$ Ts (it varies slightly for different multi-layers).

In Fig. 4 the (additional) dephasing field $1 / \tau_{\varphi}$ due to the Ni layers is plotted as a function of the Ni thickness $d_{N i}$. The three plots with full circles represent three different $\mathrm{Pb}$ layers in between the $\mathrm{Ag}$ and $\mathrm{Ni}$ films (the numbers next to the curves give the $\mathrm{Pb}$ thickness in atomic layers). For comparison the additional dephasing for $\mathrm{Ni}$ directly deposited on $\mathrm{Ag}$ is shown (full squares). Here the Ni possesses a magnetic moment already in the first mono-layer.

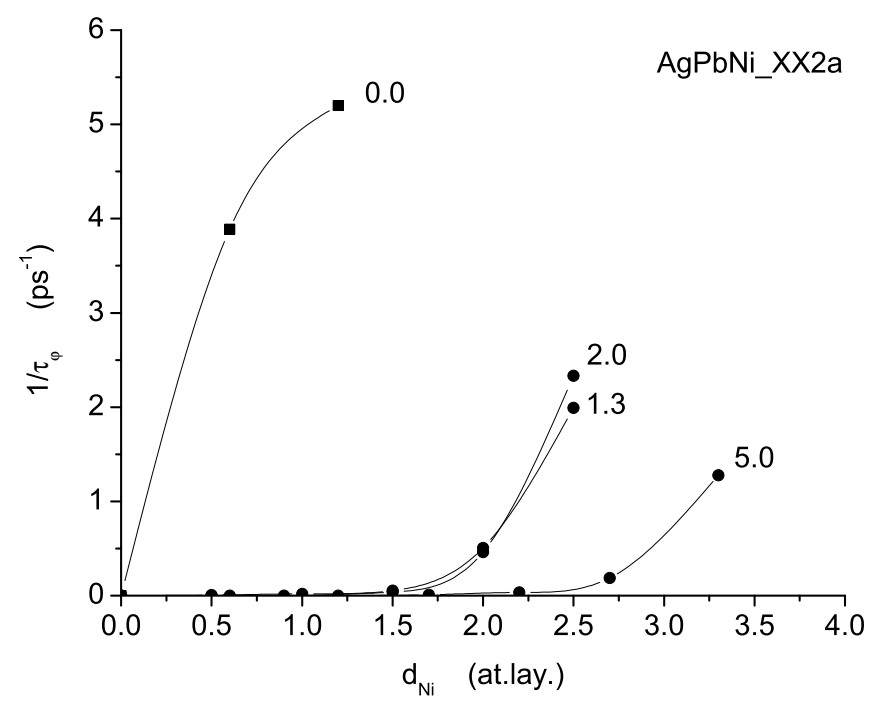

Fig.4: The additional dephasing rate $1 / \tau_{\varphi}$ of $\mathrm{AgPbNi}$ multi-layers as a function of $d_{N i}$ for different $\mathrm{Pb}$ thicknesses $d_{P b}$ (full circles). The full squares give $B_{s}$ for $\mathrm{AgNi}$ layers.

From Fig.3 and Fig.4 it is very obvious that the additional dephasing of $\mathrm{Ni}$ on $\mathrm{Pb}$ is very small for $\mathrm{Ni}$ thicknesses up to 1.5 atomic layers The dephasing rate for the $\mathrm{AgPbNi}$ multi-layer with $d_{p b}=2.0$ atomic layers and $d_{N i}=1.5$ atomic layers is $1 / \tau_{\varphi} \approx 3.0 \times 10^{10} \mathrm{~s}^{-1}$. This is the average dephasing rate for an electron that propagates within the multi-layer $\mathrm{AgPbNi}$. Since the electron spends only the fraction $d_{N i} N_{N i} /\left(d_{A g} N_{A g}+d_{P b} N_{P b}+d_{N i} N_{N i}\right)$ of the time in the $\mathrm{Ni}$ films the actual dephasing in the $\mathrm{Ni}$ film is larger by the factor $\left(d_{A g} N_{A g}+d_{P b} N_{P b}+d_{N i} N_{N i}\right) / d_{N i} N_{N i} \approx d_{A g} N_{A g} / d_{N i} N_{N i}$. This yields for the dephasing in the $\mathrm{Ni} 1 / \tau_{N i}^{w l}=3.0 \times 10^{10} s^{-1} * d_{A g} N_{A g} / d_{N i} N_{N i}$.

Moodera and Meservey interpreted the $T_{c}$-reduction in their $\mathrm{PbNi}$ double layers to be a result of magnetic scattering in the Ni film which causes a dephasing or depairing of the 
Cooper pairs. MM observed that a coverage with 1.5 atomic layers of Ni yielded a reduction of $T_{c}$ in their $\mathrm{Pb}$ film of about $\Delta T_{c} \approx 2.5 \mathrm{~K}$. This $T_{c}$-reduction is supposed to be due to a (magnetic) dephasing rate $\tau_{\varphi}$. For a weak couling superconductor a dephasing rate of $1 / \tau_{\varphi}$ yields (in linear approximation) a $T_{c}$-reduction of $k_{B} \Delta T_{c}=\pi \hbar /\left(8 \tau_{\varphi}\right)$. For the strong coupling superconductor $\mathrm{Pb}$ one obtains a correction factor of 1.4 [27] which is neglected here. This yields a dephasing rate of $1 / \tau_{\varphi} \approx 8 k_{B} \Delta T_{c} /(\hbar \pi) \approx 8 . \times 10^{11} s^{-1}$ in the double layer $\mathrm{PbNi}$ with $d_{N i}=1.5$ atomic layers As before the corresponding dephasing in the $\mathrm{Ni}$ film is larger by the factor $d_{P b} N_{P b} / d_{N i} N_{N i}, 1 / \tau_{N i}^{S U}=8 . \times 10^{11} s^{-1} d_{P b} N_{P b} / d_{N i} N_{N i}$.

Next we compare the dephasing rates in the $\mathrm{Ni}$ between the superconducting and the weak localization measurement. The ratio of the two rates is

$$
r=\frac{\left(1 / \tau_{N i}^{S U}\right)}{\left(1 / \tau_{N i}^{w l}\right)}=\frac{8 \times 10^{11} s^{-1} * d_{P b}^{M M} \gamma_{P b}}{3.2 \times 10^{10} s^{-1} * d_{A g}^{T B} \gamma_{A g}}=109
$$

Here we have replaced the ratio $N_{P b} / N_{A g}$ by the ratio of their Sommerfeld constants $\gamma_{P b} / \gamma_{A g}=4.4 . \quad d_{P b}^{M M}=9 n m$ is the Pb thickness in MM's experiment and $d_{A g}^{T B}=9 n m$ is the the Ag thickness in our experiment. Obviously the dephasing rate calculated from the reduction of $T_{c}$ in $\mathrm{Pb}$ is much larger than the observed magnetic dephasing in weak localization.

To better understand this great difference in the dephasing rates we compare the "pair propagator" in superconductivity and weak localization. Fig.5a shows the pair propagator of a Cooper pair in superconductivity. One electron with spin up moves from A to B and its time reversed partner with spin down travels from B to A. Fig.5b shows the closed loop along which two partial waves of a single electron travel in opposite directions. The fact that the path in weak localization is closed is not important for the dephasing processes. What is important is that both propagators move along time reversed paths.

Let us start with weak localization. Here the clockwise and the counter-clock wise moving electron waves interact with the environment. Electron-phonon processes, Coulomb interaction with the other electrons, and magnetic scattering cause changes in energy and phase between the two partial waves. However, there is no interaction between the two partial waves because they belong to the same electron.

In the superconducting case we have actually two electrons. Each one experiences the same interaction with the environment as in weak localization and suffers the same dephasing. For these processes the dephasing of weak localization is the same as the pair breaking in superconductivity.

But in superconductivity there are additional processes which influence the phase coherence since there are two electrons which interact. This influences their coherence in several ways: (i) the exchange of phonons supports the coherence and causes the superconductivity,(ii) due to the finite temperature the coherence of the two electrons decays with time as $\sum_{n} \exp \left[-2\left|\omega_{n}\right| t\right]\left(\omega_{n}=2 \pi k_{B} T(n+1 / 2) / \hbar=\right.$ Matsubara frequencies $)$. Since the balance of (i) and (ii) determines the $T_{c}$ of the superconductor these two processes are already taken care of.

But there can be Coulomb interaction between the two electrons which causes dephasing 
or pair weakening. This effect is not present in weak localization. The pair propagator (or pair amplitude) propagates also into the Ni film. If the two electrons of the pair hop into a d-state of a Ni atom and overlap, they repel each other and weaken the superconductivity.

If one adds transition metal atoms to a superconductor, either as impurities or as a thin film then one seriously alters the properties of the superconductor. The d-atoms possess d-resonance states. One can divide them into three groups : (i) no Coulomb interaction between d-electrons of opposite spin, (ii) a finite Coulomb interaction between d-electrons of opposite spin, but no magnetic moment, (iii) the Coulomb interaction is sufficiently strong to break the symmetry between opposite spins, and a magnetic moment develops. It is well known the third case causes pair breaking. In addition, it has been shown that not only case (ii) but also case (i) reduces the transition temperature. This phenomenon has been studied in 1970's (see for example [28], [29], [?]) and is often called pair weakening.

It might be surprising that one obtains a $T_{c}$-reduction even in the absence of Coulomb repulsion, ut its physical origin is quite simple. When a Cooper pair jumps onto two (time reversed) d-states of an d-atom their pair amplitude still decays due to the finite temperature, but it is screened from the attractive electron-phonon interaction. The fraction of the time on the d-resonances is roughly $N_{d} / N_{f e}$ where $N_{d}$ is the density of states of the d-resonance of all d-atoms while $N_{f e}$ is the density of states of the (free) conduction electrons. As a consequence the attractive interaction is reduced by a factor $\left(1-N_{d} / N_{f e}\right)$. This yields a reduction in $T_{c}$.
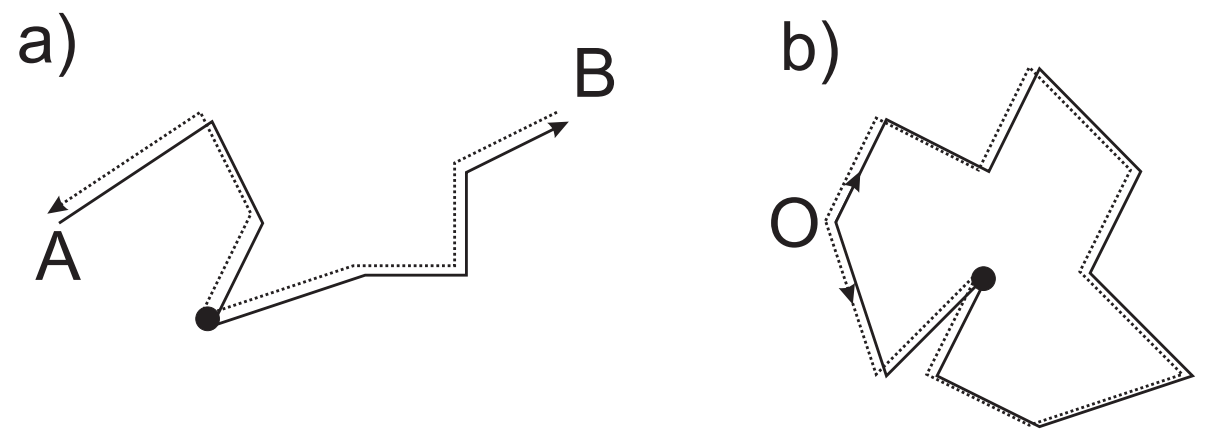

Fig.5a: The pair propagator of a Cooper pair in a superconductor.

Fig.5b: The particle-particle propagator in weak localization.

We conclude that our experimental results and those by Moodera and Meservey do not contradict each other. However, our experimental results contradict their conclusion. We find a much smaller dephasing than the reduction of $T_{c}$ would suggest. We conclude that reduction of the superconducting transition temperature is not due to dephasing by magnetic scattering but due to the resonance scattering of Cooper pairs by non-magnetic d-states. A Coulomb repulsion as studied by Friedel and Anderson will enhance the $T_{c}$-reduction.

Actually the combined investigation of magnetic dead Ni layers on top of a superconductor, the reduction of $T_{c}$, and the dephasing of weak localization would be a very effective method to study the formation of magnetic moments in d-metals. 
Acknowledgement: The research was supported by the National Science Foundation DMR-0439810. 


\section{References}

[1] G. Bergmann, Phys. Rev. Lett. 41, 264 (1978)

[2] L. Liebermann, J. Llinton, D. M. Edwards, and J. Mathon, Phys. Rev. Lett. 25, 232 (1970)

[3] D. T. Pierce and H. C. Siegmann, Phys. Rev. B9, 4035 (1974)

[4] I. Kramer, and G. Bergmann, Phys. Rev. B27, 7271 (1983)

[5] R. Meservey, P. M. Tedrow, and V. R. Kalvey, Solid state Comm. 36, 969 (1980)

[6] R. Meservey, P. M. Tedrow, and V. R. Kalvey, J. Appl. Phys. 52, 1617 (1981)

[7] B. N. Cox, R. A. Tahir-Kheli, and R. J. Elliot Phys. Rev. B 20, 2864 (1979)

[8] D. S. Wang, A. J. Freeman, and H. Krakauer, Phys. Rev. B 24, 1126 (1981)

[9] J. Tersoff and L. M. Falicov, Phys. Rev. B 26, 6186 (1982)

[10] C. Uher, R. Clarke, G. Zheng, and I. K. Schuller, Phys. Rev. B 30, 453 (1984)

[11] R. Richter, J. G. Gay, and J. R. Smith, Phys. Rev. Lett. 54, 2704 (1985)

[12] S. C. Hong, A. J. Freeman, and C. L. Fu, Phys. Rev. B 39, 5719 (1989)

[13] A. Ernst, M. Lueders, W. M. Temmerman, Z. Szotek, and G. V Laan, J. Phys. Condens. Matter 12, 5599 (2000)

[14] S. Kim, J. Jeong, J. B. Kortright, and S. Shin, Phys. Rev. B 64, 052406 (2001) 
[15] P. SanGiorgio, S. Reymond, M. R. Beasley, J. H. Kwon, and K. Char, Phys. Rev. Lett. 100,237002 (2008)

[16] O. Bourgeois, R. C. Dynes, Phys. Rev. B65, 144503 (2002)

[17] J. S. Moodera, and R. Meservey, Phys. Rev. B 29, 2943 (1984)

[18] J. S. Moodera, R. Meservey, and P. M. Tedrow, J. Appl. Phys. 55, 2502 (1984)

[19] J. S. Moodera and R. Meservey, Phys. Rev. Lett. 55, 2082 (1985)

[20] J. S. Moodera, and R. Meservey, Solid state Comm. 29, 295 (1986)

[21] J. S. Moodera and R. Meservey, Phys. Rev. B 34, 379 (1986)

[22] J. S. Moodera, and R. Meservey J. Appl. Phys. 61, 3741 (1987)

[23] G. Bergmann, Phys. Rev. B29, 6114 (1984)

[24] S. Hikami, A. I. Larkin and Y. Nagaoka, Prog. Theor. Phys. , 63, 707 (1980)

[25] P. A. Lee and T. V. Ramakrishnan, Rev. Mod. Phys. 57, 287 (1985)

[26] G. Bergmann, Physics Reports 107, 1 (1984)

[27] D. Rainer, and G. Bergmann, J. Low Temp. Phys. 14, 501 (1974)

[28] M. J. Zuckermann, Phys. Rev. 140, A899 (1965)

[29] C. F. Ratto, and A. Blandin, Phys. Rev. 156, 513 (1967)

H. Shiba, Prog. Theor. Phys. 50, 50 (1973) 